\title{
Scalable integration of nano-, and microfluidics with hybrid two-photon lithography
}

\author{
Oliver Vanderpoorten ${ }^{1,2,3}$, Quentin Peter $\mathbb{D}^{2}$, Pavan K. Challa ${ }^{2}$, Ulrich F. Keyser $\mathbb{0}^{3}$, Jeremy Baumberg ${ }^{3}$, \\ Clemens F. Kaminski ${ }^{1}$ and Tuomas P. J. Knowles ${ }^{2,3}$
}

\begin{abstract}
Nanofluidic devices have great potential for applications in areas ranging from renewable energy to human health. A crucial requirement for the successful operation of nanofluidic devices is the ability to interface them in a scalable manner with the outside world. Here, we demonstrate a hybrid two photon nanolithography approach interfaced with conventional mask whole-wafer UV-photolithography to generate master wafers for the fabrication of integrated micro and nanofluidic devices. Using this approach we demonstrate the fabrication of molds from SU-8 photoresist with nanofluidic features down to $230 \mathrm{~nm}$ lateral width and channel heights from micron to sub-100 nm. Scanning electron microscopy and atomic force microscopy were used to characterize the printing capabilities of the system and show the integration of nanofluidic channels into an existing microfluidic chip design. The functionality of the devices was demonstrated through super-resolution microscopy, allowing the observation of features below the diffraction limit of light produced using our approach. Single molecule localization of diffusing dye molecules verified the successful imprint of nanochannels and the spatial confinement of molecules to $200 \mathrm{~nm}$ across the nanochannel molded from the master wafer. This approach integrates readily with current microfluidic fabrication methods and allows the combination of microfluidic devices with locally two-photon-written nano-sized functionalities, enabling rapid nanofluidic device fabrication and enhancement of existing microfluidic device architectures with nanofluidic features.
\end{abstract}

\section{Introduction}

Micro and nanofabrication give new possibilities to analyze molecular processes with high precision. Microfluidics ${ }^{1}$ has for instance become a powerful tool to study biological systems at single cell resolution ${ }^{2-4}$ and has allowed the observation of single molecules ${ }^{5-7}$ and of single nucleation events in protein aggregation ${ }^{8}$ in a controllable environment. Microdroplet generation',

\footnotetext{
Correspondence: Tuomas P. J. Knowles (tpjk2@cam.ac.uk)

${ }^{1}$ Department of Chemical Engineering and Biotechnology, University of Cambridge, Philippa Fawcett Drive, Cambridge CB3 OAS, UK

2Department of Chemistry, University of Cambridge, Lensfield Road, Cambridge CB2 1EW, UK

Full list of author information is available at the end of the article.

These authors contributed equally: Oliver Vanderpoorten, Quentin Peter, Pavan

K. Challa
}

microfluidic diffusional sizing ${ }^{10-12}$, and electrophoresis on chip ${ }^{13-15}$ are now established techniques, but the nanofluidic regime has the potential to open up a new set of possibilities applications ${ }^{16-18}$. Many specific physical features motivate the interest in nanofluidic device fabrication, including the existence of selective transport mechanisms occurring when channel widths reach diameters close to the Debye length ${ }^{19,20}$. Interactions between protein charges and the Debye layer can be used to confine, separate, and concentrate proteins ${ }^{21}$ as well as sorting exosomes and colloids according to their size down to $20 \mathrm{~nm}^{22}$. Moreover, nanofluidics has the potential to provide increased efficiencies in blue energy, where osmotic power is harvested from Gibbs free energy of a salt gradient between two solutions connected by

\section{(c) The Author(s) 2019}

(c) (i) Open Access This article is licensed under a Creative Commons Attribution 4.0 International License, which permits use, sharing, adaptation, distribution and reproduction in any medium or format, as long as you give appropriate credit to the original author(s) and the source, provide a link to the Creative Commons license, and indicate if changes were made. The images or other third party material in this article are included in the article's Creative Commons license, unless indicated otherwise in a credit line to the material. If material is not included in the article's Creative Commons license and your intended use is not permitted by statutory regulation or exceeds the permitted use, you will need to obtain permission directly from the copyright holder. To view a copy of this license, visit http://creativecommons.org/licenses/by/4.0/. 
nanopores, nanochannels, or membranes ${ }^{23}$. Performance of reverse electrodialysis depends on the scale and geometry of the nanoscale confinement used, as has been studied $^{24,25}$ in cylindrical and conical shaped nanochannels down to tens of nanometers.

Currently, to produce lab-on-chip devices in the nanofluidic regime, electron beam lithography (EBL) and focused ion beam etching are used in clean room facilities to prototype nanochannels or nanopores in the sub$100 \mathrm{~nm}$ range in silicon/silicon nitride ${ }^{26}$. EBL can achieve channel sizes smaller than $10 \mathrm{~nm}^{27}$, but cannot pattern as fast as mask-based lithography approaches ${ }^{28}$. These approaches work well, but can be challenging to integrate with microfluidics and are costly and can require long writing times. Here, we demonstrate an approach to produce nanofluidic chips at wafer-scale in a nonclean room environment using a hybrid lithography approach bringing together direct two photon writing for defining nanoscale structures with conventional whole-wafer mask ultraviolet (UV) lithography.

The adoption of microfluidic technologies has been substantially accelerated by soft-lithography approaches ${ }^{29}$ and currently common practice for lab-on-a-chip device fabrication on a laboratory scale is UV mask photolithography followed by soft lithography. There are two main photolithographic strategies to produce master molds for soft lithography-large area mask patterning and direct laser-writing (DLW) ${ }^{30,31}$. In general, both techniques work with UV-curable photoresists such as SU-8 spincoated onto a silicon wafer, with specified thicknesses of tens of microns. Uncross-linked SU-8 is soluble in the developing agent PGMEA but becomes cross-linked and insoluble when exposed to UV radiation and post-exposure heat treatment. UV attenuating masks with transparent sections in areas to be solidified are brought between the light source and the wafer to project microfluidic chip designs onto the photoresist coated wafer. Unilluminated areas are afterwards dissolved during the development process and only the UV-exposed areas remain. The wafer surface then is used as a mold for soft lithography using PDMS casting. DLW has the advantage that there is no need for masks. In this approach a laser is scanned over the wafer and is modulated accordingly to write the intended pattern. Both of these technologies are restricted by the diffraction limit and their patterning capabilities are conventionally limited by the thickness of the photoresist between 5 and $120 \mu \mathrm{m}$ for common microfluidic fabrication because exposure triggers polymerization throughout the thickness of the photoresist. Two-photon lithography overcomes these limitations by using high power femtosecond pulse laser sources operating at twice the absorption wavelength used for the conventional DLW process. Two such high wavelength low-energy infrared radiation (IR) photons can interact with the photoinitiator molecule if they arrive within the very short lifetime of the virtual state created by the interaction of the first photon with the absorbing molecule in the photoresist ${ }^{32}$. This effect is thus strongly dependent on the power of the incident radiation $^{33}$. In two-photon lithography, polymerization only takes place when the square of the incident power reaches a threshold, unlike for conventional single photon lithography where polymerization is controlled by the intensity itself. Since the volume in which the square of the intensity is above a critical threshold can be smaller than the volume in a diffraction limited focused laser, subdiffraction limited features can be generated. Considering an additional nonlinear photo response of the photoresist materials itself and adjusting the laser intensity close to the energy threshold for polymerization, SU-8 nanorods of about $30 \mathrm{~nm}$ have previously been presented ${ }^{34}$. The fact, that two incoming photons are unlikely to interact with a photoinitiator molecule at the same time before reaching the focal spot opens up arbitrary sectioning capabilities within the spincoated photoresist layer thickness. The application range of two-photon lithography ranges from the fabrication of photonic crystals ${ }^{35}$, cell scaffolds ${ }^{36}$ to metamaterials ${ }^{37}$, biomimicry ${ }^{38}$, and additive manufactured microfluidics ${ }^{39}$. Two-photon lithography has been used ${ }^{40}$ to incorporate optical components (e.g., a total internal reflecting mirror) with a microfluidic channel using soft lithography and to demonstrate the integration of a three-dimensional microfluidic mixer into photolithographically fabricated areas on a glass coverslip ${ }^{41}$. In this paper, we enhance the strategy of combining conventional UV lithography with two-photon direct laser writing to approach the nanofluidic regime on a silicon surface. Silicon wafers are more common for microfluidic master fabrication than glass coverslips, due to their mechanical strength and surface quality. In the following, we introduce and experimentally demonstrate the successful master fabrication of nanofluidic chip devices on a silicon wafer using standard SU-8 with a channel size of $420 \mathrm{~nm}$ and show that arbitrary height channel mold fabrication down to $50 \mathrm{~nm}$ is possible. A custom-built two-photon setup was characterized with a calibration assay to determine the achievable minimal feature size of the system. Three system parameters were systematically evaluated to define the achievable resolution of the writing process: laser power, scanning speed, and focal spot offset from the wafer surface. To find the optimal values of these, test gratings were written in SU-8 at different conditions. Scanning electron microscopy (SEM) and atomic force microscopy (AFM) for the characterization of the polymerized features and the evaluated parameters, were used to incorporate nanofluidic channels into a microfluidic master. To show the successful imprinting of nanofluidic PDMS 
devices, their fluidic connectivity was demonstrated by flowing Rhodamine 6G dye through the channels and imaging their lateral width on chip, using super-resolution microscopy. The procedure presented here, fills the gap of affordable nanofabrication in biological laboratories in combination with conventional UV lithography-overcoming low patterning speeds of DLW-technology but achieving subdiffraction features in areas of interest.

\section{Results}

\section{Combining UV lithography with two-photon DLW for wafer-scale nanofluidic chip fabrication}

To explore the integration of nanofluidics with microfluidics we focus on a prototypical nano/micro device, consisting of two microfluidic channels that are connected via nanochannel junctions. These can be used for instance to isolate single molecules from a solution and study their diffusion properties. On one side a sample solution is pumped through, while the other compartment of the device will be exposed to particles or proteins that fit through the nanosized restriction connecting them.

Conventional methods to produce such nanofluidic devices are based on spincoating of sub- $100 \mathrm{~nm}$ thin photoresist films and exposure through UV attenuating masks or require electron beam lithography to push the lateral width down to the nanoscale ${ }^{42}$. Practical limitations of these methods are long writing times and variations in the photoresist thickness that render the integration of nanofluidics difficult. In our procedure presented here (see Fig. 1a-f), we can use a single spincoating process of a thick (e.g., $25 \mu \mathrm{m}$ ) SU-8 layer to fabricate nanofluidic master molds on a silicon wafer (Supplementary Figs. 1 and 2). The wafer is prebaked to remove solvent and UV-exposed through a film mask to pattern microfluidic areas on a waferscale. The wafer was then postbaked to polymerize the irradiated areas. The baking process induces a refractive index difference between exposed and unexposed areas. These refractive index edges were then used in the two-photon setup to find the regions of interest on the microfluidic master wafer, where the nanojunctions should be written. Since two-photon polymerization is induced only in the focal spot, we have arbitrary control over the height of the printed channels-we overcome the drawbacks of conventional necessary multiple spincoating steps and fabricate devices within one development process. In a second step the master wafer is developed in PGMEA, cleaned with isopropanol and dried using pressurized nitrogen. Through soft lithography, we imprinted the pattern of the functionalized nanofiltration chips in PDMS. The final PDMS imprint with additional microfluidic prefiltration section can be seen in Fig. 2a. SEM images reveal the successful connection of the two microchannels with nanochannels of $420 \mathrm{~nm}$ width and 75 microns in length (see Fig. 2b, c). Due to double exposure by UV lithography and two-photon writing the nanochannels show broader joints at the intersection with the microfluidic area.

\section{Calibration assay for micro-, to nano-scale 2-photon writing}

Since two-photon polymerization is a dose dependent process, the polymerized voxel scales with the intensity and the scanning speed of the laser beam in the photoresist. In addition, since 2PL is used here for 2.5 dimensional fabrication, the voxel truncation by the wafer surface also plays an important role on the lateral size of the written lines. A systematic approach was employed to evaluate the key factors in the system and define suitable operation parameters for micro-, to nano-sized two-photon writing on the wafer surface. Faster scanning speed as well as lower laser intensity were observed to result in a smaller voxel size. Voxel truncation influences both and is furthermore important for the height of the channel molds and proper attachment of the polymerized resin. We evaluated powers ranging from 50 to $120 \mathrm{~mW}$ measured after the acoustooptic modulator and ascending of the laser focus into the wafer from 0 to $3 \mu \mathrm{m}$ at a constant writing speed of $400 \mu \mathrm{m} /$ s. An SEM image of the calibration print can be seen in Fig. 3a. To improve SEM image quality and contrast, the sample was coated with $10 \mathrm{~nm}$ platinum. Along the vertical axis the laser power for each line was varied, where the highest intensity was used at the top line. As expected, with increasing laser power the voxel size increases from bottom to top. At the lower end the effective laser dose did not reach the polymerization energy threshold, which results in no polymerization to occur. From left to right, a decrease in lateral width can be observed due to the laser focus being lowered $3 \mu \mathrm{m}$ into the wafer over a distance of $100 \mu \mathrm{m}$. Using this calibration map, suitable parameters can be read out and the spot size adjusted according to the filling factor of the pattern-or in this case-the channel dimensions to be written. We found soft lithography compatible structures down to a size of $280 \mathrm{~nm}$ lateral width as shown by SEM imaging in Fig. 3b and even $230 \mathrm{~nm}$ if the optical design is adopted (Supplementary Fig. 3). AFM measurements of the region show heights down to $360 \mathrm{~nm}$ and are illustrated in Fig. $3 \mathrm{c}$ along with height profile measurements in Fig. 3c*. Two-photon written nanochannels are by nature of hyperbolic shape ${ }^{32}$, assuming a Gaussian intensity distribution in the focal spot, which limits this technology for the fabrication of rectangular channels. In comparison with other nanolithography techniques the achievable lateral widths are relatively large but AFM measurements at positions where the voxel is about to disappear into the sample, verified a height of down to $52 \mathrm{~nm}$ (see Fig. 3b*) and $35 \mathrm{~nm}$ height (Supplementary Fig. 4). Precise height control of the sample is required to approach fabrication in this regime due to nonlinear behavior of the channel height 

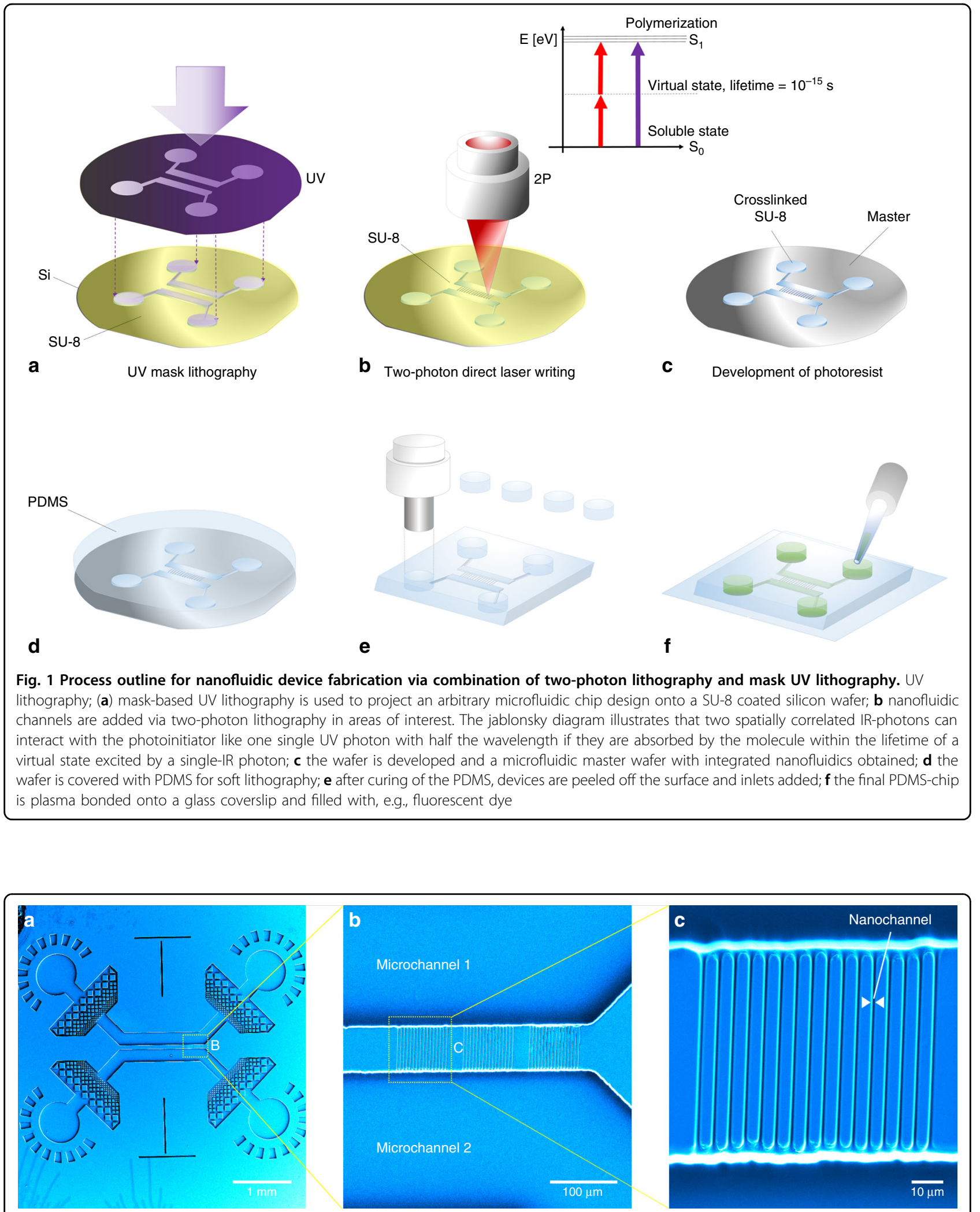

Fig. 2 Correlated SEM and AFM analysis of 2-photon nano structures. a SEM micrograph of nanofluidic PDMS imprint fabricated by the combination of UV mask lithography and 2-photon writing; $\mathbf{b}$ three nanofluidic areas with nanochannels of 75 micron length joining the two microchannels; c higher magnification shows $420 \mathrm{~nm}$ wide nanochannels imprinted in PDMS 


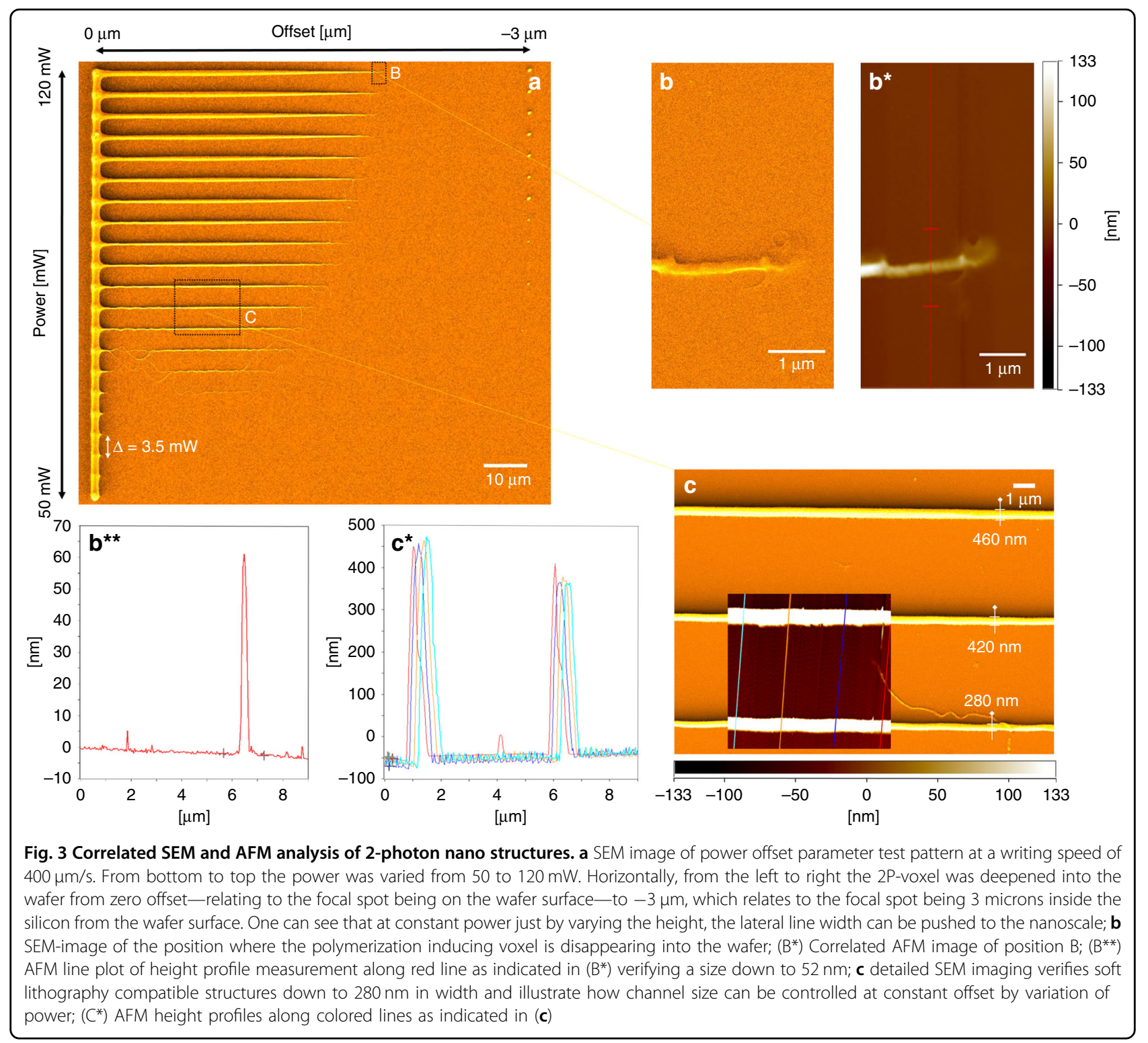

when truncating the voxel on the surface (Supplementary Fig. 5). We found that high spatial frequency components of the focused laser beam interfere above the wafer surface and result in multiple polymerization locations which can lead to detachment of the structures during the development process. Reducing the beam diameter before the objective resulted in an improvement of fabrication quality-showing similar lateral resolution and smooth lines when ascending the voxel into the wafer (Supplementary Fig. 6).

\section{TIRF-super-resolution imaging of Rhodamine $6 \mathrm{G}$ molecules in nanochannels}

In order to test the fluidic connectivity of the nanofluidic devices, we flushed from the microfluidic inlet, (A) in Fig. 4, an aqueous solution of a dye. Since the channels' dimensions are of the order or smaller than the wavelength of light, we used super resolution microscopy to image the nanofluidic channels. To this effect, total internal reflection (TIRF) illumination was used to restrict the excitation area of a fluorescence microscope to an area of approximately $100 \mathrm{~nm}$ above the coverslip. Since out of focus fluorophores are not excited, this technique has much higher signal to noise ratio and is perfectly suitable for nanochannel measurements due to spatial confinement of molecules close to the coverslip and allows the observation of single fluorescent molecules diffusing into the nanochannels from the reservoirs (Supplementary video 1). To verify the correct bonding and size consistency of the channels on chip we filled the reservoirs with Rhodamine 6G solution at femto molar 
concentration dissolved with $200 \mathrm{mM}$ MEA in phosphatebuffered saline. The solution was adjusted with $\mathrm{KOH}$ to a $\mathrm{pH}$ of 10 in order to induce blinking of the fluorescent dye molecules and enable breaking the resolution limit of conventional fluorescence microscopy by using the STORM principle ${ }^{43}$. By taking thousands of images, localizing the fluorescent emitters in each image and overlaying the data, a super resolved image can be reconstructed. A description of the setup that was used for imaging can be found in Rowlands et al. ${ }^{44}$. Single molecule localization is a useful method for the characterization of nanofluidic devices after the plasma bonding step to verify fluidic connectivity and offers an alternative to clean room equipment such as electron microscopy or AFM. From the super-resolved fluorescence microscopy image the effective channel size was measured to be $200 \mathrm{~nm}$ (full width at half maximum) (see Fig. $4 \mathrm{a}, \mathrm{b})$, and is consistent with the expected channel size read out from the calibration data acquired by SEM and demonstrates the reliability of this technique. Small distortions induced by sample handling during the bonding process can be visualized on chip and imply the importance of careful handling of the PDMS during the bonding process. The detected molecules show a Gaussian distribution along the channel width, which could be due to the increased channel height toward the nanochannel center. This caused an increase in the amount of detected molecules in the center in comparison to positions close to the walls.

\section{Discussion}

In this paper we show that two-photon lithography can be used to fabricate nanofluidic channels of arbitrary heights from micron to sub- $100 \mathrm{~nm}$ using materials conventionally employed for microfluidic fabrication and to integrate these structures with microfluidics. Two-photon lithography provides a reliable technique that decouples the influence of varying photoresist thicknesses from nanofluidic fabrication. The method reaches the upper boundary of the ultrananoscale with $35-50 \mathrm{~nm}$ channel heights, where new charge induced transport mechanisms start to appear, but remains in lateral resolution one order of magnitude larger than EBL and RIE. Also the round shape of the polymerization voxel inhibits the fabrication
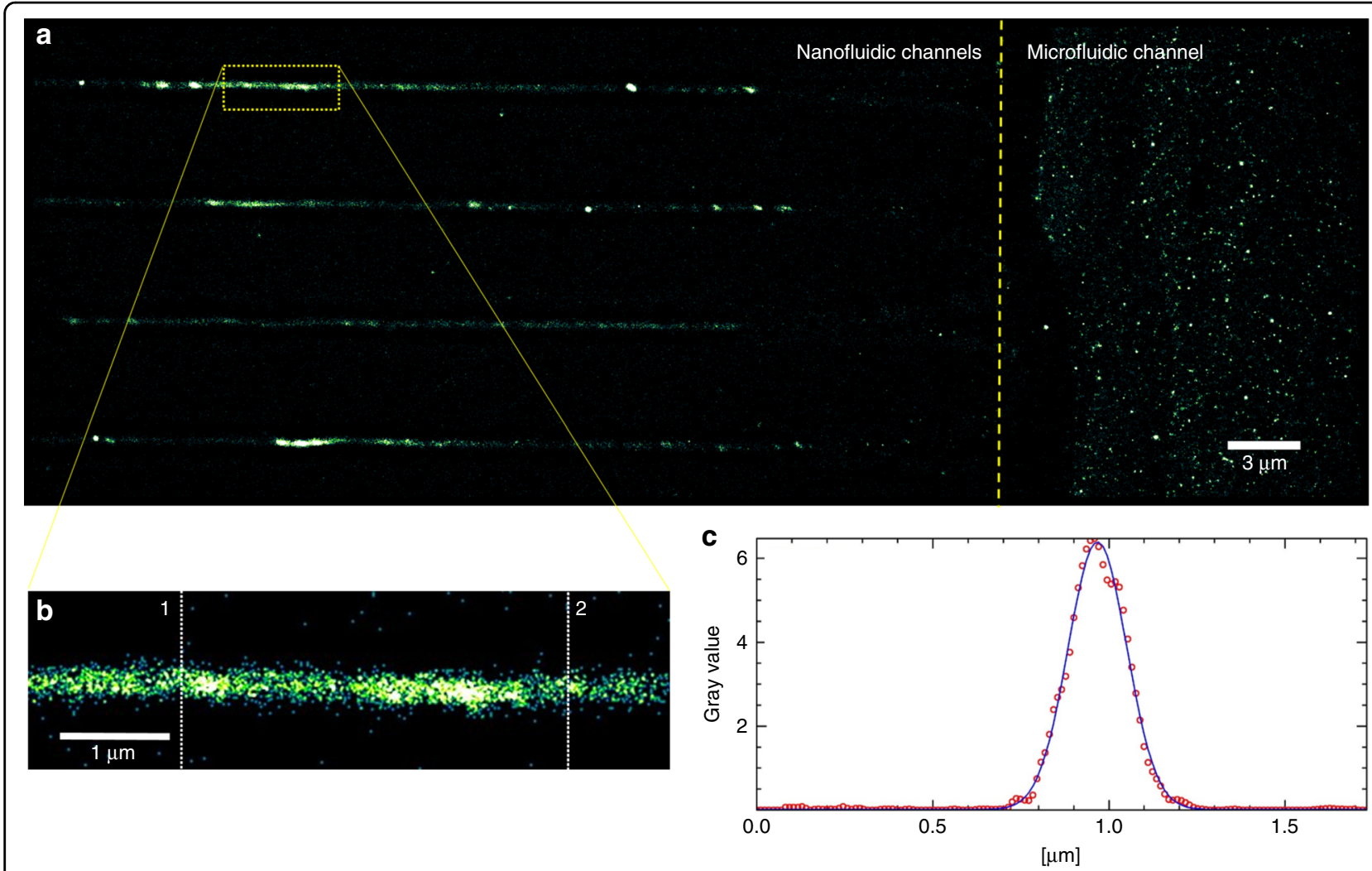

Fig. 4 Super-resolution imaging of nanofluidic devices. a Super-resolved dSTORM image of a nanofluidic PDMS chip with Rhodamine $6 \mathrm{G}$ diffusing through 2-photon written nanochannels. b Zoomed in region, verifying the successful imprinting of fluidically connected nanochannels in PDMS; the channel size was measured by averaging the vertical line profiles reaching from position 1 to position 2; $\mathbf{c}$ plot of averaged line profiles as indicated in (b) - verifying the intended channel width of $420 \mathrm{~nm}$ on chip and a FWHM of $200 \mathrm{~nm}$. Reconstruction was computed with the ThunderSTORM software plugin for ImageJ. ${ }^{45}$ 
of rectangular shaped channels which is achievable using RIE. Another technique, e.g., crack induced nanochannels - provides a simple and nonclean room technique with channel sizes in the sub- $100 \mathrm{~nm}$ regime. Approaches such as generating mechanically induced cracks in chips have a high fabrication speed and can rapidly generate nanochannels up to mm length. However, this method cannot be easily interfaced with traditional PDMS microfluidics. The combination of EBL and UV lithography is possible, but it is challenging without extending write times and costs, whereas 2PL provides high flexibility for the effective integration of arbitrary two-dimensional nanofluidic functionalities into microfluidic masters. Although twophoton lithography cannot achieve as small features as electron-beam lithography, its fabrication range is useful for a variety of biophysical and blue energy applications, e.g., charge measurements of proteins in solution using nanotraps with dimensions of $600 \mathrm{~nm}$ width and $160 \mathrm{~nm}$ height ${ }^{46}$. Nano-electroporation uses $90 \mathrm{~nm}$ wide channels for precise dose control for the injection of nanoparticles, plasmids and siRNA on a single cell basis and demonstrate the improvement of the process when channels in the submicron regime are used ${ }^{47}$. A nanofluidic power generator device of a power density of $705 \mathrm{~W} / \mathrm{m}^{2}$ using a $\mathrm{KCl}$ concentration gradient in a nanochannel of the dimensions $715 \mathrm{~nm} \times 350 \mathrm{~nm} \times 40 \mathrm{~nm}$ was demonstrated by Zhang et al. ${ }^{48}$-fabricated in a silicon chip. However, care must be taken when comparing softlithography devices with silicon applications. Handling the PDMS carefully during the bonding process is crucial to not bend or collapse nanostructures on the final substrate. Silicon chips are more reliable and stable during operation, but PDMS is cost-effective and offers the advantage of fast replacement. The lateral resolution of the two-photon effect can be pushed to the limit by using the STED principle. ${ }^{49}$ Using photoresists consisting of tri- and tetraacrylates and 7 diethylamino-3-thenoylcoumarin and surrounding the focal spot with a depletion beam pushes the polymerized features size to $55 \mathrm{~nm}$ at a resolution of $120 \mathrm{~nm}$, which shifts two-photon lithography technology further towards EBL resolution for nanofabrication.

\section{Methods}

\section{Sample preparation and development}

In all, $25 \mu \mathrm{m}$ of SU-8 were spin coated (BRUKER) at $3000 \mathrm{rpm}$ onto a silicon wafer. The wafer was soft baked and treated according to the protocol of the photoresist distributor (Microchem). A microfluidic mask pattern was then projected onto the photoresist for $50 \mathrm{~s}$ with the setup described in Challa et al. ${ }^{50}$. The wafer post baked at $95^{\circ} \mathrm{C}$ so that the interfaces between developed and undeveloped regions become visible. After the two-photon writing process the whole wafer was again baked at $95^{\circ} \mathrm{C}$ and finally rinsed with PGMEA and IPA.

\section{Two-photon lithography setup}

To produce the high intensities needed for two photon excitation to occur, a Menlo System C-Fiber $780 \mathrm{HP}$ Er: doped fiber oscillator with a repetition rate of $100 \mathrm{MHz}$ with integrated amplifier and second harmonic generation was used as light source of the two photon system. The laser pulse width is $120 \mathrm{fs}$. The setup is optimized to produce nanoscale features on a $100 \mu \mathrm{m} \times 100 \mu \mathrm{m}$ field. The beam is expanded to fill the whole back aperture of the objective lens (Leica, PL APO, Magn. 100×, NA = 1.4, oil) using a Thorlabs beam expander (AR coated: 650-1050 nm, GBE05-B). To make positioning of the focal spot over a whole $3^{\prime \prime}$ wafer possible, two PI linearprecision stages (M-404.2PD, Ball screw, $80 \mathrm{~mm}$ wide, ActiveDrive) were mounted perpendicular on top of each other with a suitable adaptor plate, connected to two individual PI Mercury DC Motor Controllers (C-863.11, 1 Channel, wide range power supply). On top, a PI NanoCube with a travel range of $100 \mu \mathrm{m} \times 100 \mu \mathrm{m} \times 100 \mu \mathrm{m}$ is mounted for high precision movement (pos. resolution: $1 \mathrm{~nm}$ ) of the sample during the writing process. We focus on the wafer/photoresist interface using the backreflection of the laser beam at low intensity (where polymerization will not occur), and capture the image via a USB-camera ( $\mu$ Eye ML, Industry camera, USB 3.0) with a tube lens (Thorlabs AC 254-100-A-ML, BBAR coating $400-700 \mathrm{~nm}, f=100.0 \mathrm{~mm}$ ) mounted above the objective. The objective is mounted onto a Thorlabs Z812B stage to allow wider travel range in $z$ when moving over the wafer. The laser modulation is controlled by an acousto-optic modulator by AA Optoelectronics mounted after the laser output port connected to a fast switching power supply (ISOTECH,DC power supply, IPS 33030). In order to provide an open-source setup all the control programming is realized in Python as well as the automated writing of a calibration assay of the system.

\section{Conclusion}

We integrated successfully nanofluidic functionalities into microfluidic devices by combining two-photon lithography with mask based UV lithography on a silicon wafer. Pre-exposed areas generated first through conventional UV lithography undergo a refractive index change which can then be used to align the two-photon writing process. We showed that the two-photon lithography setup presented here is capable of producing features down to $230 \mathrm{~nm}$ lateral width on a silicon wafer surface in SU-8 photoresist and successfully integrated $420 \mathrm{~nm}$ wide nanochannels into a microfluidic master design. By ascending progressively the exposure voxel, reliable nanochannel molds of sub $100 \mathrm{~nm}$ in height were fabricated. This regime has the potential to produce PDMS devices that are comparable to EBL or RIE-etched chips. In contrast to other techniques e.g nanochannel 
fabrication by cracking, where mechanics and reagents define the shape of the formed nano junctions - twophoton lithography allows the integration of arbitrary nano-sized patterns and complex shapes including varying channel sizes into microfluidic devices. We verified the reliability of the fabrication process by comparing SEM images of a SU-8 calibration sample with TIRF fluorescence super-resolution imaging in the final PDMS devices. Further improvements in resolution of the process could be achieved by a change in photoresist composition or post-processing of the photoresist via temperature and plasma treatment to thin out written structures. We hope to provide with this method a fast, reliable and flexible pathway for nanofluidic device fabrication to enable readily the addition of nanofluidic features to conventional devices.

\section{Acknowledgements}

This work was supported by the Engineering and Physical Sciences Research Council [Grant numbers EP/L015889/1 and EP/L027151/1], the European research Council, the Winton Program for the Physics of Sustainability and the Newman Foundation. The authors would also like to thank the NanoDTC for additional funding and the Maxwell Community for scientific support. This project has received funding from the European Union's Horizon 2020 research and innovation program under Grant agreement No. 674979-NANOTRANS. The work was partially funded by Horizon 2020 program through 766972-FET-OPEN-NANOPHLOW. U.F.K. acknowledges funding from an ERC Consolidator Grant (DesignerPores 647144).

\section{Author details}

'Department of Chemical Engineering and Biotechnology, University of Cambridge, Philippa Fawcett Drive, Cambridge CB3 OAS, UK. ${ }^{2}$ Department of Chemistry, University of Cambridge, Lensfield Road, Cambridge CB2 1EW, UK. ${ }^{3}$ Cavendish Laboratory, Department of Physics, University of Cambridge, J. J. Thomson Avenue, Cambridge CB3OHE, UK

\section{Authors' contributions}

O.V. and P.C. were conducting the experiments, were involved in the SEM and AFM imaging of calibration samples, master wafers, and PDMS stamps, as well as device fabrication and interpreted the data from SEM and AFM imaging together with Q.P. O.V. imaged the nanochannel devices using TIRF microscopy and was improving the device fabrication procedure. P.C. was project initiator and main contributor to the optical design and hardware considerations of the 2PL system. Q.P. developed open-source software in Python to control the 2PL setup and was involved in the automation of the process and its optimization using all available actuators. All authors provided input into the paper.

\section{Conflict of interest}

The authors declare that they have no conflict of interest.

Supplementary information accompanies this paper at https://doi.org/ 10.1038/s41378-019-0080-3.

Received: 7 February 2019 Revised: 26 May 2019 Accepted: 25 June 2019 Published online: 09 September 2019

\section{References}

1. Whitesides, G. M. The origins and the future of microfluidics. Nature $\mathbf{4 4 2}$ 368-373 (2006). arXiv:1011.1669v3.

2. Klein, A. M. et al. Droplet barcoding for single-cell transcriptomics applied to embryonic stem cells. Cell 161, 1187-1201 (2015).
3. Macosko, E. Z. et al. Highly parallel genome-wide expression profiling of individual cells using nanoliter droplets. Cell 161, 1202-1214 (2015).

4. Croote, D., Darmanis, S., Nadeau, K. C. \& Quake, S. R. Antibodies cloned from single. Science 1309, 1306-1309 (2018).

5. Huang, S., Romero-Ruiz, M., Castell, O. K., Bayley, H. \& Wallace, M. I. Highthroughput optical sensing of nucleic acids in a nanopore array. Nat. Nanotechnol. 10, 986-991 (2015).

6. Bell, N. A. \& Keyser, U. F. Digitally encoded DNA nanostructures for multiplexed, single-molecule protein sensing with nanopores. Nat. Nanotechnol. 11, 645-651 (2016).

7. Chen, K. et al. Digital data storage using DNA nanostructures and solid-state nanopores. Nano Lett. 19, 1210-1215 (2019).

8. Janasek, D., Franzke, J. \& Manz, A. Scaling and the design of miniaturized chemical-analysis systems. Nature $\mathbf{4 4 2}, 374$ (2006).

9. Knowles, T. P. J. et al. Observation of spatial propagation of amyloid assembly from single nuclei. Proc. Natl Acad. Sci. 108, 14746-14751 (2011).

10. Challa, P. K. et al. Real-time intrinsic fluorescence visualization and sizing of proteins and protein complexes in microfluidic devices. Anal. Chem. 90, 3849-3855 (2018). PMID: 29451779.

11. Yates, E. V. et al. Latent analysis of unmodified biomolecules and their complexes in solution with attomole detection sensitivity. Nat. Chem. 7, 802-809 (2015).

12. Bortolini, C. et al. Resolving protein mixtures using microfluidic diffusional sizing combined with synchrotron radiation circular dichroism. Lab. Chip 19, 50-58 (2019).

13. Herling, T. W. et al. A microfluidic platform for real-time detection and quantification of protein-ligand interactions. Biophys. J. 110, 1957-1966 (2016).

14. Saar, K. L. et al. On-chip label-free protein analysis with downstream electrodes for direct removal of electrolysis products. Lab Chip 18, 162-170 (2018).

15. Saar, K. L., Müller, T., Charmet, J., Challa, P. K. \& Knowles, T. P. J. Enhancing the resolution of micro free flow electrophoresis through spatially controlled sample injection. Anal. Chem. 90, 8998-9005 (2018). 8b01205 PMID: 29938505.

16. Bocquet, L. \& Charlaix, E. Nanofluidics, from bulk to interfaces. Chem. Soc. Rev 39, 1073-1095 (2010).

17. Marbach, S., Dean, D. S. \& Bocquet, L. Transport and dispersion across wiggling nanopores. Nat. Phys. 14, 1108-1113 (2018).

18. Jubin, L., Poggioli, A., Siria, A. \& Bocquet, L. Dramatic pressure-sensitive ion conduction in conical nanopores. Proc. Natl Acad. Sci. 115, 4063-4068 (2018).

19. Schoch, R. B., Han, J. \& Renaud, P. Transport phenomena in nanofluidics. Rev. Mod. Phys. 80, 839-883 (2008).

20. de la Escosura-Muñiz, A. \& Merkoçi, A. Nanochannels for electrical biosensing Trends Anal. Chem. 79, 134-150 (2016).

21. Inglis, D. W., Goldys, E. M. \& Calander, N. P. Simultaneous concentration and separation of proteins in a nanochannel. Angew. Chem. Int. 50, 7546-7550 (2011).

22. Wunsch, B. H. et al. Nanoscale lateral displacement arrays for the separation of exosomes and colloids down to $20 \mathrm{~nm}$. Nat. Nanotechnol. 11, 936-940 (2016).

23. Siria, A., Bocquet, M. L. \& Bocquet, L. New avenues for the large-scale harvesting of blue energy. Nat. Rev. Chem. 1, p 6 (2017).

24. He, Y. et al. Electrokinetic analysis of energy harvest from natural salt gradients in nanochannels. Sci. Rep. 7, 1-15 (2017).

25. Kim, D. K., Duan, C., Chen, Y. F. \& Majumdar, A. Power generation from concentration gradient by reverse electrodialysis in ion-selective nanochannels. Microfluid. Nanofluidics 9, 1215-1224 (2010).

26. Yusko, E. C. et al. Controlling protein translocation through nanopores with bio-inspired fluid walls. Nat. Nanotechnol. 6, 253-260 (2011).

27. Bruno, G. et al. Unexpected behaviors in molecular transport through sizecontrolled nanochannels down to the ultrananoscale. Nat. Commun. 9, 1-10 (2018).

28. Del Campo, A. \& Greiner, C. SU-8: A photoresist for high-aspect-ratio and 3D submicron lithography. J. Micromech. Microeng. 17, p 92 (2007).

29. Xia, Y. \& Whitesides, G. M. Soft lithography. Annu. Rev. Mater. Sci. 28, 153-184 (1998). 1111.6189v1.

30. Feng, R. \& Farris, R. J. Influence of processing conditions on the thermal and mechanical properties of SU8 negative photoresist coatings. J. Micromech. Microeng. 13, 80-88 (2003).

31. Park, S. H., Yang, D. Y. \& Lee, K. S. Two-photon stereolithography for realizing ultraprecise three-dimensional nano/microdevices. Laser Photonics Rev. 3, 1-11 (2009).

32. Lee, K. S., Kim, R. H., Yang, D. Y. \& Park, S. H. Advances in 3D nano/microfabrication using two-photon initiated polymerization. Prog. Polym. Sci. 33, 631-681 (2008). 
33. Malinauskas, M., Farsari, M., Piskarskas, A. \& Juodkazis, S. Ultrafast laser nanostructuring of photopolymers: a decade of advances. Phys. Rep. 533, 1-31 (2013).

34. Juodkazis, S., Mizeikis, V., Seet, K. K., Miwa, M. \& Misawa, H. Two-photon lithography of nanorods in SU-8 photoresist. Nanotechnology 16, 846-849 (2005).

35. Thiel, M., Rill, M. S., Von Freymann, G. \& Wegener, M. Three-dimensional bichiral photonic crystals. Adv. Mater. 21, 4680-4682 (2009).

36. Richter, B. et al. Guiding cell attachment in 3D microscaffolds selectively functionalized with two distinct adhesion proteins. Adv. Mater. 29 https://doi. org/10.1002/adma.201604342 (2017).

37. Rill, M. S. et al. Gold helix photonic metamaterial as broadband circular polarizer. Science 325, 1513-1515 (2009).

38. Marino, A. et al. A 3D real-scale, biomimetic, and biohybrid model of the blood-brain barrier fabricated through two-photon lithography. Small 14, 1-9 (2018).

39. Hengsbach, S. \& Lantada, A. D. Rapid prototyping of multi-scale biomedical microdevices by combining additive manufacturing technologies. Biomed. Microdevices 16, 617-627 (2014).

40. Eschenbaum, C. et al. Hybrid lithography: combining UV-exposure and two photon direct laser writing. Opt. Express 21, 29921 (2013).

41. Lin, Y., Gao, C., Gritsenko, D., Zhou, R. \& Xu, J. Soft lithography based on photolithography and two-photon polymerization. Microfluid. Nanofluid. 22, 97 (2018).
42. Kovarik, M. L. \& Jacobson, S. C. Nanofluidics in lab-on-a-chip devices. Anal. Chem. 81, 7133-7140 (2009).

43. Dempsey, G. T., Vaughan, J. C., Chen, K. H., Bates, M. \& Zhuang, X. Evaluation of fluorophores for optimal performance in localization-based super-resolution imaging. Nat. Methods 8, 1027-1040 (2011). NIHMS150003.

44. Rowlands, C. J., Ströhl, F., Ramirez, P. P., Scherer, K. M. \& Kaminski, C. F. Flat-field super-resolution localization microscopy with a low-cost refractive beamshaping element. Sci. Rep. 8, 1-8 (2018).

45. Ovesný, M., Ǩrížek, P., Borkovec, J., Švindrych, Z. \& Hagen, G. M. ThunderSTORM: a comprehensive ImageJ plug-in for PALM and STORM data analysis and super-resolution imaging. Bioinformatics 30, 2389-2390 (2014).

46. Ruggeri, F. et al. Single-molecule electrometry. Nat. Nanotechnol. 12, 488-495 (2017).

47. Boukany, P. E. et al. Nanochannel electroporation delivers precise amounts of biomolecules into living cells. Nat. Nanotechnol. 6, 747-754 (2011).

48. Zhang, Y., Huang, Z., He, Y. \& Miao, X. Enhancing the efficiency of energy harvesting from salt gradient with ion-selective nanochannel. Nanotechnology https://doi.org/10.1088/1361-6528/ab0ed8 (2019).

49. Wollhofen, R., Katzmann, J., Hrelescu, C., Jacak, J. \& Klar, T. A. 120nm resolution and 55nm structure size in STED-lithography. Opt. Express 21, 10831 (2013).

50. Challa, P. K., Kartanas, T., Charmet, J. \& Knowles, T. P. J. Microfluidic devices fabricated using fast wafer-scale LED-lithography patterning. Biomicrofluidics 11, 014113 (2017) 